\title{
Effect of bars in AGN host galaxies and black hole activity
}

\author{
M. S. Alonso ${ }^{1,2}$, G. Coldwell ${ }^{1,2}$, and D. G. Lambas ${ }^{1,3}$ \\ ${ }^{1}$ Consejo Nacional de Investigaciones Científicas y Técnicas, Argentina \\ e-mail: salonso@icate-conicet.gob.ar \\ 2 ICATE, CONICET-UNSJ, CC 49, 5400 San Juan, Argentina \\ 3 IATE, CONICET, OAC, Universidad Nacional de Córdoba, Laprida 854, X5000BGR Córdoba, Argentina
}

Received 27 July 2012 / Accepted 12 November 2012

\begin{abstract}
Aims. With the aim of assessing the effects of bars on active galactic nuclei (AGN), we present an analysis of host characteristics and nuclear activity of active galaxies with and without bars.

Methods. We selected AGN host galaxies from the Sloan Digital Sky Survey Data Release 7 (SDSS-DR7), using the available emission-line fluxes. By visual inspection of SDSS images we classified the face-on spiral hosts of AGNs brighter than $g$-mag $<16.5$ into barred or unbarred. With the purpose of providing an appropriate quantification of the effects of bars, we also constructed a suitable control sample of unbarred active galaxies with similar redshift, magnitude, morphology, bulge sizes, and local environment distributions.

Results. We find that the bar fraction, with respect to the full sample of spiral face-on AGN host galaxies, is $28.5 \%$, in good agreement with previous works. barred AGN host galaxies show an excess of young stellar populations (as derived with the $D_{n}(4000)$ spectral index), dominated by red $u-r$ and $g-r$ colors, with respect to the control sample, suggesting that bars produce an important effect on galaxy properties of AGN hosts. Regarding the nuclear activity distribution, we find that barred active galaxies show a shift toward higher Lum[OIII] values with respect to AGN without bars. In addition, we also find that this trend is more significant in less massive, younger stellar population and bluer AGN host galaxies. We found that the fraction of powerful AGN increases toward more massive hosts with bluer colors and younger stellar populations residing in denser environments. However, barred host AGN systematically show a higher fraction of powerful active nuclei galaxies with respect to the control sample. We also explored the accretion rate onto the central black holes, finding that barred AGN host show an excess of objects with high accretion rate values with respect to unbarred active galaxies.
\end{abstract}

Key words. galaxies: active - galaxies: peculiar - galaxies: spiral - galaxies: formation - galaxies: evolution - Galaxy: nucleus

\section{Introduction}

It is widely accepted that the mechanism responsible for active galactic nuclei (AGN) is based on the accretion of matter by massive black holes at the center of galaxies (Lynden-Bell 1969; Rees 1984). Several theories have been proposed to explain the black hole feeding mechanisms needed to transport mass to the inner most region of the central engine. The major galaxy interaction (Alonso et al. 2007), minor mergers (Roos 1981, 1985; Hernquist \& Mihos 1995), tidal disruption produced by galaxy harassment (Lake et al. 1998), gas dynamic processes involving the presence of multiple black holes (Begelman et al. 1980), and gravitational instabilities in the disk of barred galaxies (Shlosman et al. 1990) can cause gas transfer to the central regions of galaxies. One must also consider that it is plausible that at any given time or for any given AGN type, more than one mechanism plays a role.

In particular, bars play an important role in the evolution, star-formation, and nuclear activity of galaxies. The relation between star-forming galaxies and bars has been the subject of several works (Balzano 1983; Devereux 1987), finding that in some cases bars seem to be an appropriate way to produce radial gas flow, which favors an increasing star formation rate in the galaxies (Martin 1995) that could be even more effective than galaxy interactions (Ellison et al. 2011). Considering the known connection between star formation and nuclear activity of galaxies
(Springel et al. 2005; Di Matteo et al. 2005), it can be expected that bars also increase the AGN activity.

Furthermore, several studies have suggested that the gas clouds within galaxies suffer shocks by interaction with the edges of the bars, producing angular momentum losses and allowing a flow of gas within the innermost regions of galaxies (Shlosman et al. 1990). Also, a system of bars ("bars within bars") of different lengths and properties has been proposed (Shlosman et al. 1990) and observed (Emsellem et al. 2001) to have an association with the AGN activity. Erwin (2004) found fifty double-barred galaxies whose inner or secondary bar is embedded within a large-scale, outer or primary bar, of the catalog of 67 barred galaxies that contain elliptical stellar structures inside their bars. In addition, bars can explain the formation of bulges, which are also directly related to the probability of occurrence of AGN (Wyse 2004). Coelho \& Gadotti (2011) analyzed barred and unbarred galaxy samples with similar stellar mass distributions, finding similar age-metallicity diagrams in both samples; however, the distribution of bulge ages in barred galaxies showed an excess of younger populations with respect to bulges in unbarred ones. These authors also found, for lowmass bulges, twice as many AGN in barred galaxies compared to unbarred galaxies.

However, although there is observational evidence that bars cause central concentrations of gas compared to non-barred galaxies, there is no clear agreement whether the large-scale 
bars are efficient for the transport of material directly to the regions closest to the black hole (Knapen 2005). Ho et al. (1997) found that the existence of bars in a sample of 300 spiral galaxies has no significant effect on the AGN power. More recently, Oh et al. (2012) found a signal of bar effect in the AGN activity mainly present in blue galaxies with low black hole mass. This AGN sample was selected with the Kauffmann et al. (2003) criteria from a volume limited sample of SDSSDR7 late-type galaxies $(b / a>0.7)$ with good image qualities. On the other hand, Lee et al. (2012a) used a sample of AGN selected with the Kewley et al. (2001) classification, also from the SDSS-DR7 volume limited sample of SDSS-DR7 late-type galaxies $(b / a>0.6)$, finding that AGN power is not enhanced by bars. In this analysis, Lee et al. (2012a) did not include the composite galaxies within the sample of AGN hosts. These galaxies are, in general, younger and less massive (Kewley et al. 2006) than the AGN sample defined by the Kewley et al. (2001) criteria. Accordingly, the different AGN selection criteria may affect the results related to the effect of enhanced AGN activity by bars.

This discrepancy in the results could be due mainly to two points. i) A biased selection of the control sample, used for the comparison of the obtained results. In this sense, Perez et al. (2009) suggested that a control sample for interacting galaxies should be selected cohere, at least, redshift, morphology, stellar masses, and local densities, match these criteria are, also, applicable to the building of control samples of barred galaxies because they have different properties than unbarred galaxies. ii) The short life time of bars of 1-2 Gyr (Bournaud et al. 2005) which represents a brief period in the life of an AGN. Moreover, studies using numerical simulations (Bournaud \& Combes 2002) found that bars within galaxies can be destroyed and re-formated several times during the galaxy life. Moreover, 3D N-body simulations (Athanassoula et al. 2005) showed that the central mass concentration necessary to destroy the bar is, at least, several per cent of the mass of the disk, suggesting that supermassive black holes are not likely to destroy pre-existing bars. Therefore, bars formed at different times, and with different conditions, might be present in the sample of barred galaxies.

In this work we studied in detail the properties of barred host galaxies and the effects that bars have on the AGN power for a sample of spiral galaxies, comparing the results with a carefully selected sample of unbarred AGN host. In Sect. 2 we describe the type II AGN selection method, the bar classification, $z$ and the control sample selection criteria. In Sect. 3 we analyse the dependence of the AGN host galaxy properties on the bar presence. The influence of bars on the black hole activity is detailed in Sect. 4, and finally the conclusions are summarized in Sect. 5. The adopted cosmology through out this paper is, $\Omega=0.3, \Omega_{\lambda}=0.7$ and $H_{0}=100 \mathrm{~km} \mathrm{~s}^{-1} \mathrm{Mpc}$.

\section{The AGN SDSS-DR7 catalog}

The Sloan Digital Sky Survey (SDSS, York et al. 2000) is the largest galaxy survey at the present. It uses a $2.5 \mathrm{~m}$ telescope (Gunn et al. 2006) to obtain photometric and spectroscopy data that will cover approximately one-quarter of the celestial sphere and collect spectra of more than one million objects. The seventh data release imaging (SDSS-DR7, Abazajian et al. 2009) comprises 11663 square degrees of sky imaged in five wave-bands $(u, g, r, i$ and $z$ ) containing photometric parameters of 357 million objects. SDSS-DR7 therefore represents the final data set that will be released with the original targeting and galaxy selection (Eisenstein et al. 2001; Strauss et al. 2002). The main galaxy sample, which contains $\sim 700000$ galaxies with measured spectra and photometry, is essentially a magnitude-limited spectroscopic sample (Petrosian magnitude) $r_{\text {lim }}<17.77$, most of galaxies span a redshift range $0<z<0.25$ with a median redshift of 0.1 (Strauss et al. 2002).

Several physical galaxy properties have been derived and published for the SDSS-DR7 galaxies: gas-phase metallicities, stellar masses, indicators of recent major star-bursts, current total and specific star-formation rates, emission-line fluxes, Sérsic index, etc. (Brinchmann et al. 2004; Tremonti et al. 2004; Blanton et al. 2005). These data were obtained from the $\mathrm{MPA} / \mathrm{JHU}^{1}$ and the $\mathrm{NYU}^{2}$ value added catalogs.

For the AGN selection we used the publicly available emission-line fluxes. The method for emission-line measurement is detailed in Tremonti et al. (2004). Additionally, we corrected the emission-line fluxes for optical reddening using the Balmer decrement and the Calzetti et al. (2000) obscuration curve. We assumed an $R_{V}=A_{V} / E(B-V)=3.1$ and an intrinsic $\mathrm{H} \alpha / \mathrm{H} \beta=3.1$. Since the true uncertainties in the emissionline measurements were underestimated, the signal-to-noise ratio $(\mathrm{S} / \mathrm{N})$ of every line was calculated with the emission-line flux errors adjusted according to the uncertainties suggested by the MPA/JHU catalog 3 .

The active galaxy sample was selected using a standard diagnostic diagram proposed by Baldwin et al. (1981, hereafter BPT). This diagram allows for the separation of type II AGN from normal star-forming galaxies using emission-line ratios and depending on their position in the diagram. Furthermore, we used only galaxies with an $S / N>2$ for all lines intervening in the diagnostic diagram used to distinguish AGN from HII galaxies. This $\mathrm{S} / \mathrm{N}$ cut was selected taking into account that the adjusted uncertainties almost duplicated the original errors. Therefore, taking into account the relation between spectral lines, [OIII] $\lambda 5007, \mathrm{H} \beta$, [NII] $\lambda 6583$, and $\mathrm{H} \alpha$, within the BPT diagram we followed the Kauffmann et al. (2003) criterion to select type II AGN as those with,

$\log _{10}([\mathrm{OIII}] / \mathrm{H} \beta)>0.61 /\left(\log _{10}([\mathrm{NII} / \mathrm{H} \alpha])-0.05\right)+1.3$.

\subsection{Selection of barred AGN host galaxies}

With the aim of obtaining an AGN sample with barred galaxy hosts, we first cross-correlated the SDSS-DR7 active galaxies with the spiral objects obtained from the Galaxy Zoo catalog (Lintott et al. 2011), which comprises a morphological classification of nearly 900000 galaxies drawn from the SDSS. Then, we restricted the active spiral galaxy sample in redshift $(z<0.1)$ and imposed a magnitude cut such as the extinction corrected SDSS $g$-mag is brighter than 16.5. We selected galaxies with axial ratio $b / a>0.4$ since the efficiency of the visual classification decreases with galaxy inclination. With these restrictions, our AGN sample in the SDSS-DR7 comprises 6772 galaxies.

By using the photometric SDSS-DR7, we classified the host galaxies of the active spiral galaxy sample morphologically taking into account the naked-eye detection of barred features, finding 1927 AGN hosted in barred galaxies. This represents a fraction of $28.5 \%$ with respect to the full sample of 6772 AGN in spiral host galaxies, and this value agrees with the bar fraction found by visual inspection of optical galaxy samples in previous works. We excluded 207 objects that did not completely match

\footnotetext{
http://www .mpa-garching.mpg.de/SDSS/DR7/

http://sdss.physics.nyu.edu/vagc/

http://www .mpa-garching.mpg.de/SDSS/DR7/raw_data. html
} 
Table 1. Results of classification.

\begin{tabular}{lcc}
\hline \hline Classification & Number of galaxies & Percentages \\
\hline Barred & 1927 & $28.5 \%$ \\
Unclear barred & 207 & $3.0 \%$ \\
Unbarred & 4638 & $68.5 \%$ \\
\hline Total & 6772 & $100.0 \%$ \\
\hline
\end{tabular}

the bar classification. The details of the classification are listed in Table 1. We checked for possible dependencies of our classification on redshift by computing the fraction of barred hosts in different redshift intervals, finding a nearly constant value of $\sim 30 \%$. In particular, there are 244 galaxies within $z<0.02$ of which $74(30 \%)$ have bars. Consequently, the small bars visible in this closer subsample, are not expected to be a significant fraction in our sample of unbarred objects.

Several studies, carried out by means of visual inspection of different optical galaxy samples (e.g. the RC3 and UGC, de Vaucouleurs et al. 1991; Nilson 1973; Marinova et al. 2009) found a bar fraction of 25-30\%. Moreover, Masters et al. (2010) computed the mean bar fraction, finding $29.4 \%$ of barred galaxies from a sample of 13665 Galaxy Zoo disk objects. More recently, Oh et al. (2012) detected a bar fraction of $51.2 \%$ (715 AGN barred galaxies) in the sample of active galaxies obtained from SDSS-DR7 in the redshift range $0.01<z<0.05$. Also, other studies using near-infrared images from 2MASS (Menéndez-Delmestre et al. 2007) and SDSS image decompositions of $g, r$ and $i$ bands (Gadotti 2009) found a higher percentage of bars, $59 \%$ and $42 \%$, respectively.

To corroborate the accuracy of our selection criteria, we cross-correlated our sample with barred AGN host galaxies taken from the catalog of Nair \& Abraham (2010). These authors constructed a catalog of detailed visual classification for 14034 galaxies in the Sloan Digital Sky Survey Data Release 4 (SDSS-DR4). The sample includes galaxies in the redshift range $0.01<z<0.1$, with $g-$ mag $<16$. They detected a total of 454 barred active galaxies sub-classified, depending on the relatively light contribution of the bar in to strong, intermediate, and weak. We found 399 (87.9\%) barred active galaxies common in the two samples. However, the Nair \& Abraham (2010) catalog classified 307 strong and intermediate barred AGN host galaxies from the total sample from which we found 297 common galaxies, representing the $96.5 \%$ of overlap. Considering that we matched catalogs derived through visual classification, and that this kind of classification could be subjective, the level of agreement is very high.

We focus our attention on the effects of bars on the nuclear activity. It is very well known that galaxy interactions contribute to the AGN power enhancement (Alonso et al. 2007). Accordingly, for the purpose of having unbiased results with respect to the bar effects in AGN, we derived a sample of AGN hosted by relatively isolated barred galaxies. To construct a sample with a suitable isolation criterion for barred galaxies, we required that any neighboring galaxy within a region of $500 \mathrm{kpc}^{-1}$ in projected separation and $\Delta V>1000 \mathrm{~km} \mathrm{~s}^{-1}$ must be fainter than the barred active galaxy. With this restriction, the final sample of isolated barred AGN is composed of 1530 galaxies.

\subsection{Control sample}

To provide a suitable quantification of the effects of bars on active galactic nuclei, we constructed a control sample of unbarred
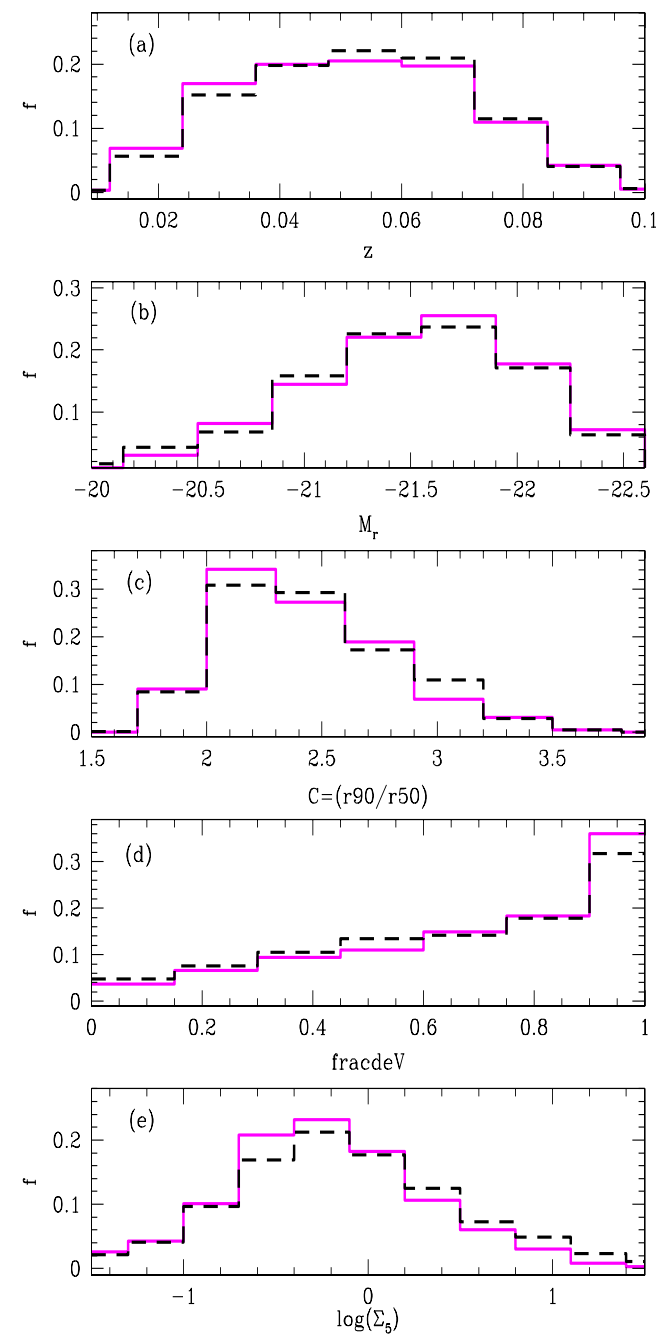

Fig. 1. Distribution of redshift, luminosities, concentration index, bulge size indicator, and local density parameter, $z, M_{r}, C$, fracdeV, and $\log \left(\Sigma_{5}\right)(a, b, c, d$, and $e$ panels), for barred AGN host galaxies (solid lines) and active galaxies without bars in the control sample (dashed lines).

AGN hosts to compare this with the barred host results. First, from the sample of 4638 unbarred AGN host spiral face-on galaxies (with $b / a>0.4$ and $g$-mag $<16.5$ ) we selected isolated objects using a suitable isolation criterion defined in the previous section for the barred AGN host sample. Then, we defined a sample of control galaxies using a Monte Carlo algorithm that selects galaxies in the unbarred isolated sample, with similar redshift, $r$-band magnitude, and concentration index ${ }^{4}, C$, distributions of the barred AGN host sample (see panels $a, b$ and $c$ in Fig. 1). We restricted the control sample of unbarred AGN hosts to match the concentration parameter $C$ of the AGN barred sample to obtain a similar bulge to disk ratio in both samples. This means that the possible differences in the results are associated with the presence of bars and not with the difference in the global morphology.

Coelho \& Gadotti (2011) found that bulges in barred galaxies have a lower mass than in unbarred galaxies with a similar total stellar mass distribution. Since this tendency could affect our future analysis, we considered galaxies with similar bulge prominence in both samples and selected the control galaxies

4 $C=r 90 / r 50$ is the ratio of Petrosian $90 \%-50 \%$ r-band light radii. 


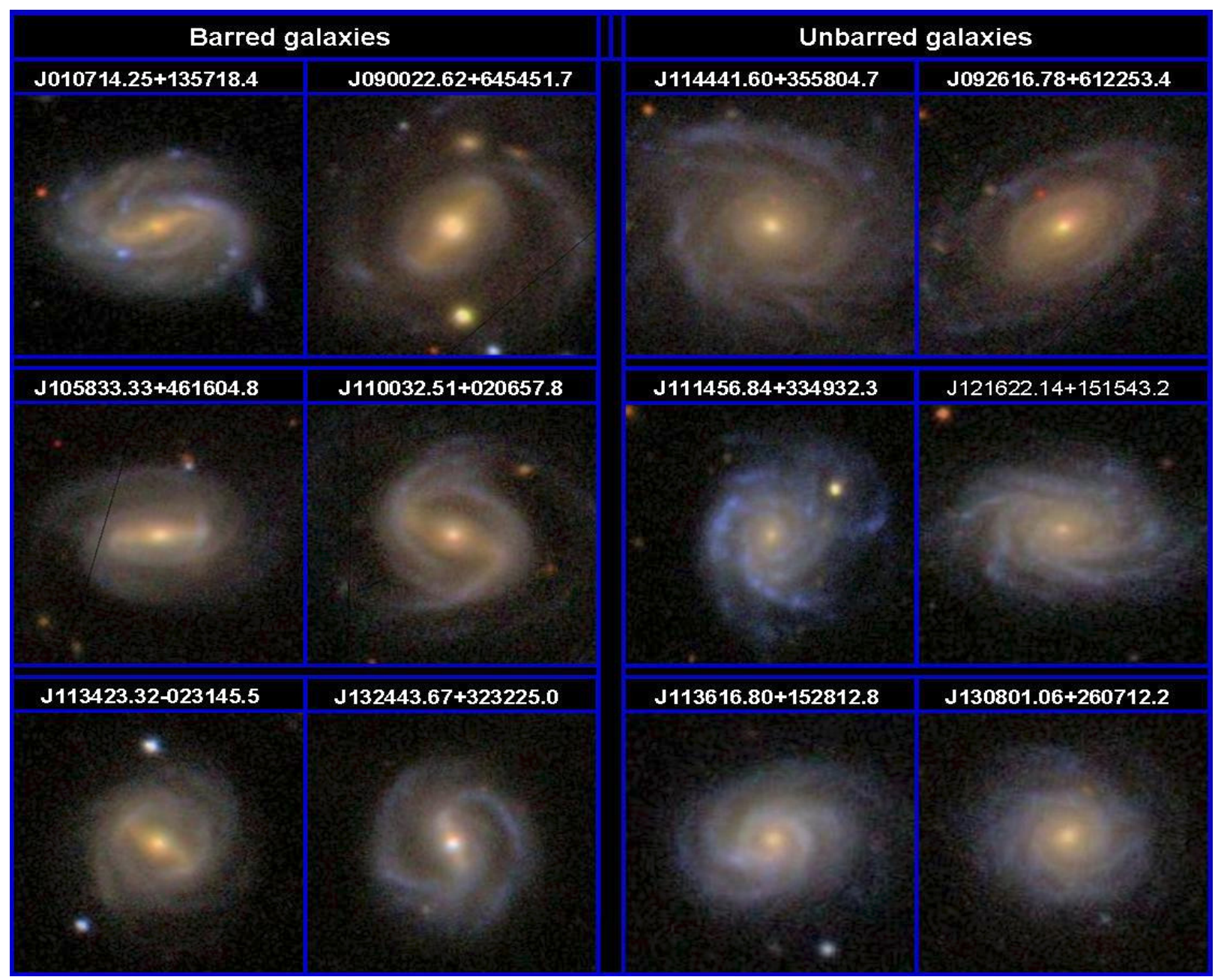

Fig. 2. Images of typical examples of barred and unbarred active galaxies in our samples. The image sizes are $35 \times 35 \mathrm{kpc}^{2}$.

with a similar distribution of the fracdeV parameters as for the barred AGN host sample (panel $d$ in Fig. 1). The SDSS fracdeV parameter is a good indicator of bulge sizes in galaxies with disk morphology (Kuehn 2005; Bernardi et al. 2010; Masters et al. 2010; Skibba et al. 2012) and Masters et al. (2010) concluded that this result is independent of the presence of bars.

We also selected a control sample with a similar distribution of the local density environment to that of barred galaxies. With this aim, for both isolated samples (barred and unbarred) we defined a projected local density parameter, $\Sigma_{5}$. This parameter is calculated through the projected distance $d$ to the fifth nearest neighbor, $\Sigma_{5}=5 /\left(\pi d^{2}\right)$. Neighbors were chosen to have luminosities above a certain threshold and with a radial velocity difference of less than $1000 \mathrm{~km} \mathrm{~s}^{-1}$. We also imposed the condition $M_{r}<-20.5$ for our selection in the SDSS (Balogh et al. 2004). Panel (e) in Fig. 1 shows the $\log \left(\Sigma_{5}\right)$ distribution for both samples.

The procedure followed to construct this control catalog ensures that it will have the same selection effects as the barred AGN host catalog, and consequently, it can be used to estimate the actual difference between active galaxies with and without bars, unveiling the effect of the bars on the nuclear activity driven by the central black hole feeding.

Figure 2 shows images of typical examples of barred and unbarred AGN host galaxies (left and right panels) selected for this work.

\section{AGN host properties}

Different studies have shown that the bars can induce several processes that modify many properties of the galaxies (e.g. Sellwood \& Wilkinson 1993; Combes et al. 1993; Zaritsky et al. 1994) and also can have associated different star formation history. The bars can trigger nuclear starbursts (Gadotti \& dos Anjos 2001), modify the galactic structure (Athanassoula 1983; Buta \& Combes 1996), change the chemical composition and dynamics of gas (Martin \& Roy 1994; Combes et al. 1993), etc. With the purpose of analyzing the impact of bars on the AGN host properties, in this section we explore the effect of bars on the stellar population, color index, and local density environment of the AGN host galaxies.

In Fig. 3 (upper panels) we show the $(u-r)$ and $(g-r)$ color distributions of barred and unbarred active galaxies. Evidently, that there is a clear excess of barred AGN hosts with redder colors. In the same direction, recent results of Masters et al. (2010) found that passive red spiral galaxies had a high fraction of bars, within a sample obtained from the Galaxy Zoo catalog. Furthermore, Oh et al. (2012) also found that a significant number of barred galaxies are redder than typical late-type galaxies, and the majority of unbarred spiral galaxies are located in the blue peak. Our results on barred AGN host galaxies are consistent with those of barred galaxies without nuclear activity found in previous works. 
Table 2. Percentages of red colors and young stellar population for barred AGN host galaxies, with respect to unbarred AGN host in the control sample.

\begin{tabular}{lccc}
\hline \hline Restrictions & $(u-r)>2.3$ & $(g-r)>0.72$ & $D_{n}(4000)<1.7$ \\
\hline \% of barred & $58.1 \%$ & $63.6 \%$ & $30.1 \%$ \\
\% of unbarred & $49.3 \%$ & $54.2 \%$ & $22.3 \%$ \\
\hline
\end{tabular}
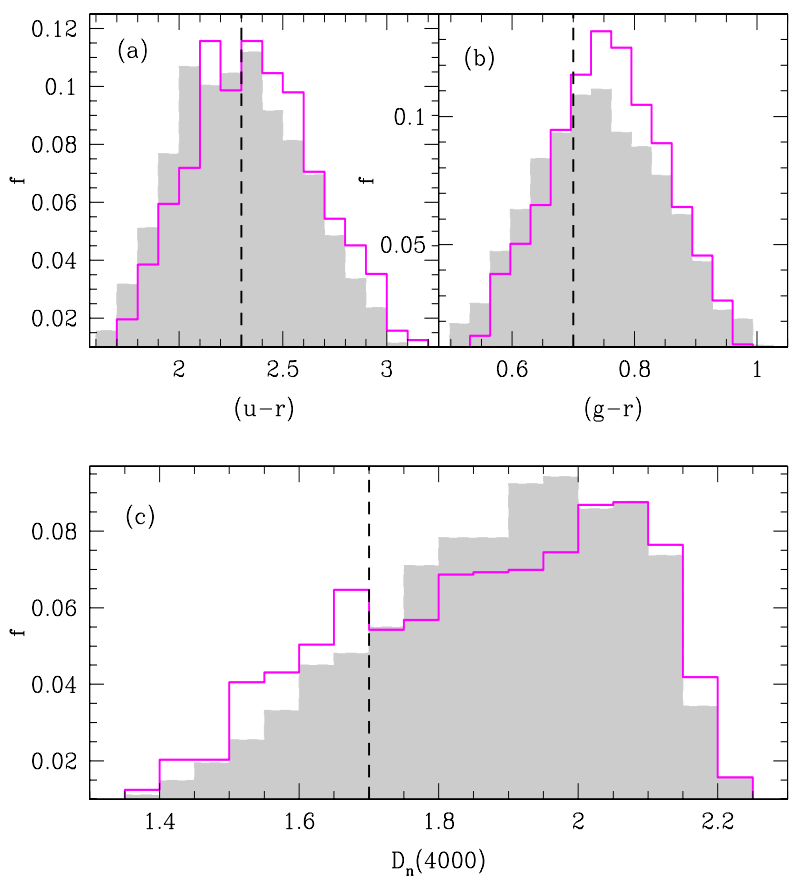

Fig. 3. Distribution of colors, $(u-r)$ and $(g-r)$, (panels a) and b)) and stellar age indicator, $D_{n}(4000)$, c) panel for barred AGN host galaxies (solid lines) and AGN host galaxies without bars (shaded histogram).

To complement this analysis, we also used the spectral in$\operatorname{dex} D_{n}(4000)$ as an indicator of the age of stellar populations. This spectral discontinuity occurring at $4000 \AA$ (Kauffmann et al. 2003) arises by an accumulation of a large number of spectral lines in a narrow region of the spectrum, an effect that is important in the spectra of old stars. We adopted Balogh et al. (1999) definition of $D_{n}(4000)$ as the ratio of the average flux densities in the narrow continuum bands (3850-3950 and $4000-4100 \AA)$. In the lower panel of Fig. 3 we show the distribution of $D_{n}(4000)$ values for barred AGN host and galaxies without bars in the control sample. We find an excess of barred objects exhibiting lower values of $D_{n}(4000)$, showing that the hosts of barred AGN host galaxies have a significant young stellar population. The differences in the colour and $D_{n}(4000)$ distributions of barred and unbarred AGN host (Fig. 3) were confirmed using the Kolmogorov-Smirnov test with a significance higher than $99.95 \%$.

According to these results, we defined a population of red and young hosts by restricting the sample to values of colors and $D_{n}(4000)$ parameters for the barred AGN host sample. The thresholds are indicated by the dashed vertical line in Fig. 3. In Table 2 we quantify this excess of red colors and young stellar population of barred AGN host galaxies with respect to the control sample.

To understand the behavior of the colors and stellar populations of barred AGN host with respect to active galaxies without bars, we analyzed the relative fractions of red and young stellar

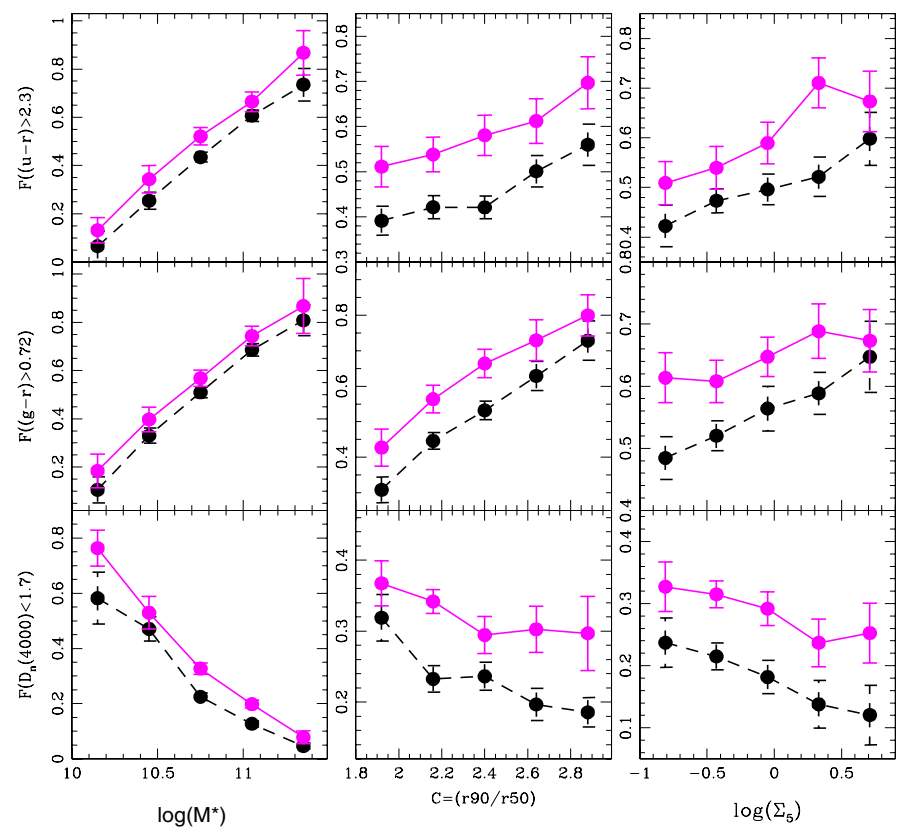

Fig. 4. Fraction of galaxies with $(u-r)>2.3$ (upper panel), $(g-r)>$ 0.73 (middle panel) and $\left(D_{n}(4000)\right)<1.7$ (lower panel), as a function of the stellar mass, concentration index, and local environment parameter, $\log \left(\Sigma_{5}\right)$, (left, middle and right panels), for barred and unbarred AGN host galaxies (solid and dashed lines).

populations. In Fig. 4 we show the fraction of $u-r>2.3$ (upper panel), $g-r>0.72$ (middle panel) and $D_{n}(4000)<1.7$ (lower panel) as a function of stellar mass, $\log \left(M^{*}\right)$, concentration index, $C$, and local environment parameter, $\log \left(\Sigma_{5}\right)$, for barred AGN host galaxies and for the control sample (solid and dashed lines). Errors were estimated by applying the bootstrap resampling technique in all figures (Barrow et al. 1984), the error-bar length correspond to $1 \sigma$. As can be seen in the left panels, the fraction of red AGN host galaxies increases toward high stellar masses and, on the other hand, the fraction of young stellar population decreases with $\log \left(M^{*}\right)$. Clearly barred AGN host galaxies have a systematically higher fraction of redder colors and younger stellar population in different bins of stellar mass, suggesting that bars have a significant effect in producing a rapid transformation of gas into stars associated to reddening and a young population of stars.

The middle panels of Fig. 4 show that the red/young fraction increase/decrease toward the more concentrated host galaxies for both barred and unbarred AGN host. This result is consistent with expectations, since galaxies with higher values of the concentration index are related to the bulge type morphology, and lower concentration objects to spiral galaxies. It can also be seen that barred AGN host galaxies (solid lines) show higher fractions of red and young population for the whole range of the $C$ parameter compared to their counterparts of unbarred AGN host in the control sample.

For the relation between bars and environment, different works find that local density might be important in the formation of bars, although results are contradictory. Numerical simulations (e.g. Walker et al. 1996; Mihos et al. 1997; Berentzen et al. 2004) show that bars are created by interactions between galaxies. Different observational studies find that the bar galaxy fraction does not depend on the environment (van den Bergh 2002; Mendez-Abreu et al. 2010; Martinez \& Muriel 2011). More recently, Lee et al. (2012b) also found that the fraction of weak 
bars has no correlation with environmental parameters, suggesting that there is no direct evidence for environmental stimulation of bar formation. On the other hand, Elmegreen et al. (1990) show a correlation between the bar fraction and environment for spiral galaxies, finding the highest fraction in pair systems. More recently, Skibba et al. (2012) clearly detected the environmental dependence of barred galaxies, in a sample obtained from the Galaxy Zoo 2 project, such that barred galaxies tend to be found in denser environments than their unbarred counterparts, with environmental correlations that are statistically significant (at a level of $>6 \sigma$ ) on scales of $150 \mathrm{kpc}$ to a few Mpc. The authors argued that the small number statistics of previous studies inhibited a detection of a bar-environment correlation.

To study the dependence of colors and stellar populations of AGN hosts on the environment, we show in the right panels of Fig. 4 the red, young population fractions of barred AGN hosts in the control sample as a function of the local galaxy density parameter $\left(\Sigma_{5}\right)$. A weak trend consistent with the expected increase of the red fractions with local galaxy density for AGN hosts with and without bars is evident. Nevertheless, the fraction of low $D_{n}$ hosts, where a significant young stellar population is present, decreases towards higher values of $\Sigma_{5}$. Interestingly, in the whole range of $\log \left(\Sigma_{5}\right)$, the red and young population fractions of galaxies in barred AGN host galaxies (solid lines) exceeds that of unbarred AGN host in the control sample (dashed lines). According to the boostrap error bars, this signal is statistically meaningful at more than $1 \sigma$-level in the left panels, and at a $2 \sigma$-level in the middle and right panels.

From these analysis we conclude that the dependence of colors and stellar populations on environment suggests that bar perturbations are a suitable physical mechanism for a fast star formation activity and a stirring of dust in the central regions of these AGN host galaxies.

\section{Black hole activity}

As a tracer of the AGN activity, we focus here on the dustcorrected luminosity of the [OIII] $\lambda 5007$ line, Lum[OIII]. This estimator is widely used by several authors (Mulchaey et al. 1994; Kauffmann et al. 2003; Heckman et al. 2004, 2005; Brinchmann et al. 2004) and some catalogs of [OIII] $\lambda 5007$ luminosities have been published (Whittle 1992; Xu et al. 1999) The main reason is that the $[\mathrm{OIII}]$ line is particularly appealing because it is one of the strongest narrow emission lines in optically obscured AGN and has a very low contamination from contributions of star formation in the host galaxy. AGN hosts are typically massive galaxies, therefore their metallicities are expected to be high due to the mass-metallicity relation (Kauffmann et al. 2003; Tremonti et al. 2004). For our sample, the majority of the galaxies have $M^{*}>10^{10} M_{\odot}$ so that higher metallicities are expected. Kauffmann et al. (2003) showed from an inspection of the BPT diagrams that high-metallicity hosts have a low contamination in the Lum[OIII] from star formation. Furthermore, Heckman et al. (2004) computed the average contribution of the AGN to the Lum[OIII] for a sample of composite galaxies (AGN above the Kauffmann et al. (2003) criterium but below the Kewley et al. (2001) 2001 line in the BPT diagram) and the AGN-dominated galaxies (above the Kewley et al. (2001) 2001 demarcation in the BPT diagram) taken from the SDSS. The results of this work indicate that the Lum[OIII] emission from composite galaxies comes from AGN activity in a range of $50 \%$ to $90 \%$, while for AGN-dominated galaxies, more than $90 \%$ of the Lum[OIII] comes from AGN emission. In addition, Heckman et al. (2005) found a correlation between the

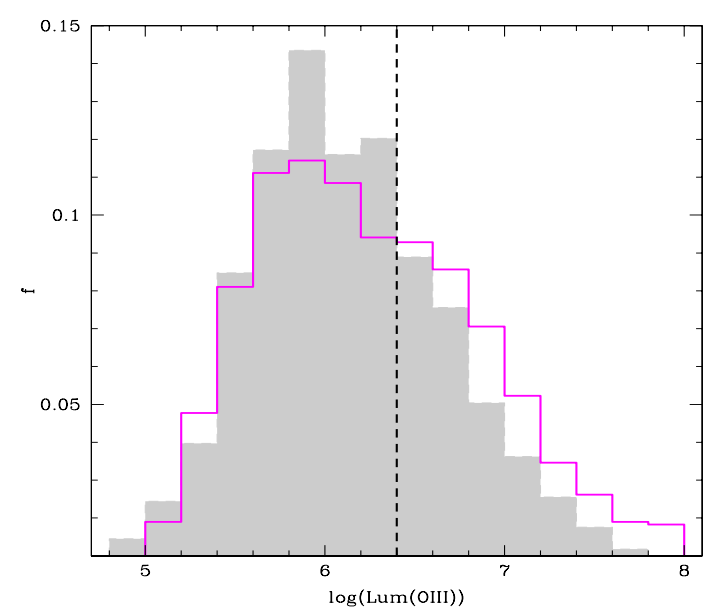

Fig. 5. Distribution of $\log (\operatorname{Lum}[\mathrm{OIII}])$ for barred active galaxies (solid line) and unbarred AGN host (full surfaces).

hard X-ray emission of AGN and the OIII luminosity, concluding that at low redshifts, the selection according to optical emission lines will recover most AGN selected according to their hard X-ray emission.

The influence of the bars on nuclear activity can be seen in Fig. 5 in the histogram of $\log (\operatorname{Lum}[\mathrm{OIII}])$ for barred AGN host (solid line) and galaxies in the control sample (full surface). Clearly, barred active galaxies show an excess of high Lum[OIII] values with respect to the control sample. The KolmogorovSmirnov statistics allow us to quantify the difference between these distributions with a significance of $99.99 \%$. Here, we consider the luminosity $\mathrm{Lum}[\mathrm{OIII}]=10^{6.4} L_{\odot}$ as a limit between weak and powerful AGN by selecting values that represent the excess of nuclear activity for the barred AGN host sample (43.4\% for barred active galaxies versus $31.5 \%$ for the control sample). This threshold is represented by the dashed vertical line in Fig. 5.

Left and middle panels in Fig. 6 show the nuclear activity distributions, $\log (\operatorname{Lum}[\mathrm{OIII}])$, of barred active galaxies compared with the unbarred AGN host counterparts. The analysis was performed for the different ranges of stellar masses, $\log (M *)$, concentration parameter, $C$, stellar age indicator, $D_{n}(4000),(g-r)$ color, and local environment, $\log \left(\Sigma_{5}\right)$. We find that in more massive galaxies with older stellar populations and red colors, the Lum[OIII] distributions of barred AGN host are similar to those of AGN without bars in the control sample. Interestingly, in less massive younger stellar population and bluer host galaxies $\left(\approx M^{*}<10^{10.7} M_{\odot}, D_{n}(4000)<1.8\right.$ and $(g-r)<0.75)$, the nuclear activity distributions of barred and unbarred AGN host exhibit significant differences, with barred objects having a higher percentage of powerful active nuclei galaxies. This behavior can be interpreted in terms of the greater ability of bars to fuel the central black holes in galaxies with a higher gas fraction expected in these hosts. Moreover, we explored the nuclear activity distributions of barred and unbarred AGN host with different concentration parameter. The critical concentration index value of $C=2.5$ was adopted to segregate concentrated, bulge-like $(C>2.5)$ galaxies from more extended, disc-like $(C<2.5)$ objects. Bearing this in mind, we find that in AGN hosts with higher values of $C<2.5$, indicating bulge type morphology, barred galaxies show a higher fraction of powerful AGN compared with those of unbarred AGN host in the control sample. 
M. S. Alonso et al.: Effect of bars in AGN host galaxies and black hole activity
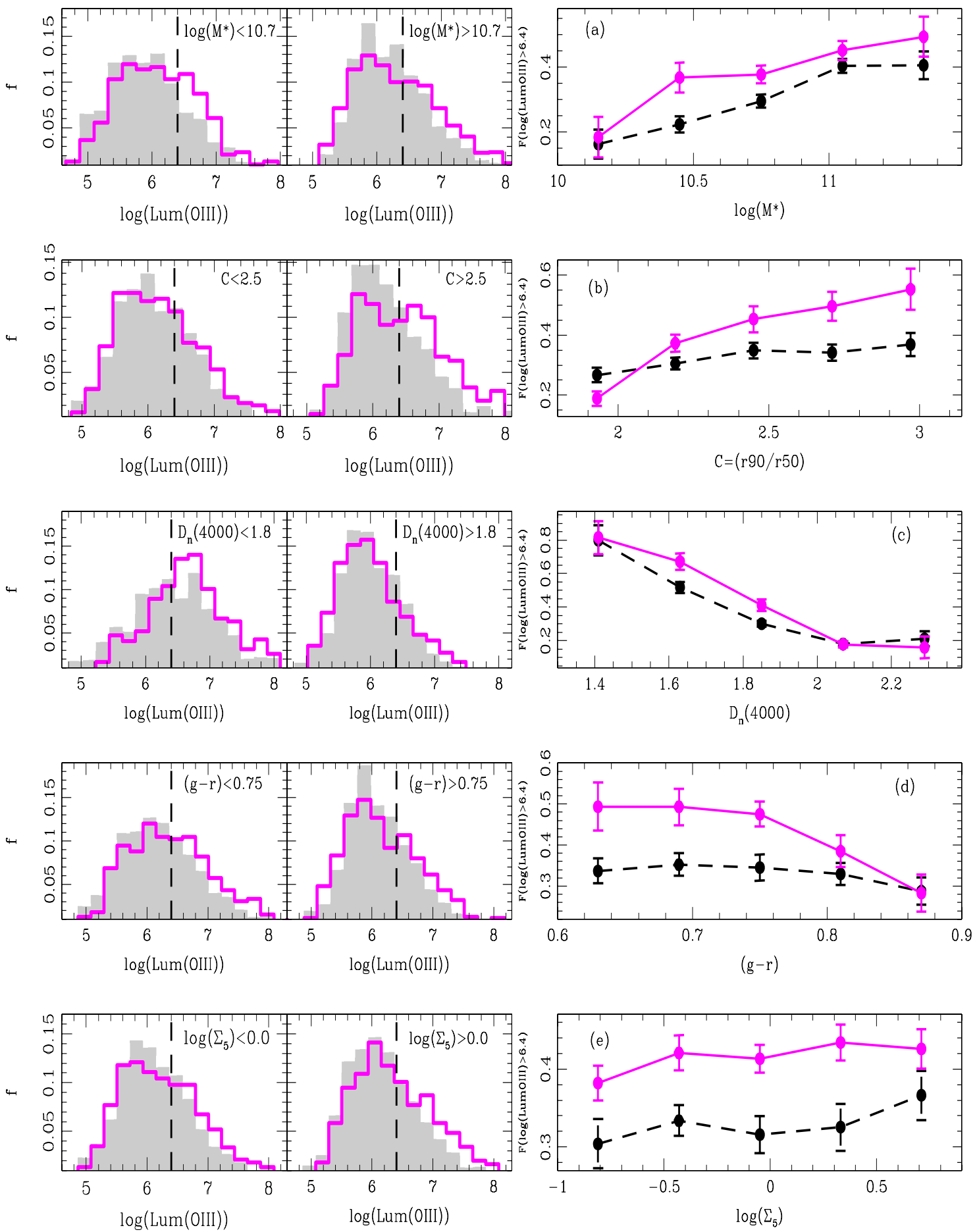

Fig. 6. Distributions of $\log (\operatorname{Lum}[\mathrm{OIII}])$ for barred and unbarred AGN host galaxies (solid lines and full surfaces) in different ranges of log $\left(M^{*}\right)$, $C, D_{n}(4000),(g-r)$ and $\log \left(\Sigma_{5}\right)$. Right panels: show the fraction of galaxies with $\log (\operatorname{Lum}[\mathrm{OIII}])>6.4$ as a function of stellar masses a), concentration parameter b), stellar age population $\mathbf{c}),(g-r)$ color $\mathbf{d})$, and local density environment $\mathbf{e})$ for barred AGN host galaxies and unbarred AGN host (solid and dashed lines).

We also selected two different local environment ranges, and there are clearly no differences in the nuclear activity distributions. In addition, in Table 3 we list the percentage of barred and unbarred active galaxies with $\log (\operatorname{Lum}[\mathrm{OIII}])>6.4$, in different ranges of stellar masses, $C$ index, age populations, $(g-r)$ colors, and $\Sigma_{5}$ parameter. These percentages reflect the results of Lum[OIII] distributions (Fig. 6), confirming that most powerful AGN tend to inhabit blue and young barred host galaxies but with a higher value in the concentration index.
To support the results obtained with these distributions, we present in the right panels of Fig. 6 the fraction of galaxies with $\log (\operatorname{Lum}[\mathrm{OIII}])>6.4$ as a function of galaxy properties. Panel a) shows the fraction of galaxies with high Lum[OIII] values as a function of stellar mass content of the corresponding host galaxies. This relation was calculated for barred AGN host and for active galaxies in the control sample without bars. The results in Fig. 6a clearly show that, in general, most massive hosts show a higher fraction of AGN with Lum[OIII $]>10^{6.4} L_{\odot}$. 
Table 3. Percentages of barred and unbarred active galaxies with $\log (\operatorname{Lum}[\mathrm{OIII}])>6.4$ in different ranges of stellar masses, $C$, $D_{n}(4000),(g-r)$, and $\log \left(\Sigma_{5}\right)$.

\begin{tabular}{lcc}
\hline \hline Ranges & \% of barred AGN host & $\%$ of unbarred AGN host \\
\hline $\log \left(M^{*}\right)>10.7$ & $43.5 \%$ & $36.6 \%$ \\
$\log \left(M^{*}\right)<10.7$ & $35.5 \%$ & $22.9 \%$ \\
\hline$C<2.5$ & $35.1 \%$ & $31.5 \%$ \\
$C>2.5$ & $51.8 \%$ & $34.4 \%$ \\
\hline$D_{n}(4000)>1.8$ & $24.8 \%$ & $22.5 \%$ \\
$D_{n}(4000)<1.8$ & $66.3 \%$ & $51.1 \%$ \\
\hline$(g-r)>0.75$ & $36.3 \%$ & $29.1 \%$ \\
$(g-r)<0.75$ & $42.3 \%$ & $35.9 \%$ \\
\hline $\log \left(\Sigma_{5}\right)>0.0$ & $44.3 \%$ & $32.6 \%$ \\
$\log \left(\Sigma_{5}\right)<0.0$ & $40.1 \%$ & $32.8 \%$ \\
\hline
\end{tabular}

We can also see that barred AGN host objects systematically show a higher fraction of powerful active galaxies irrespective of the stellar mass content, indicating an enhancement of the black hole activity or barred AGN host with respect to AGN residing in unbarred hosts. In a similar way, Oh et al. (2012) found that the AGN strength is enhanced by the presence of a bar and linearly correlates with stellar mass.

In panel b) we show the fraction of powerful active galaxies as a function of the concentration index, $C$. In this plot we find that the fraction of barred active galaxies with $\log (\operatorname{Lum}[\mathrm{OIII}])>$ 6.4 increases toward higher $C$ values, while the fraction of powerful unbarred AGN host appear to remain almost constant with the morphological parameter. In addition, barred active galaxies show, on average, a higher fraction of powerful AGN with respect to the control sample, but with a decrease of powerfully barred AGN host for the lowest values of the $C$ parameter where both barred and unbarred AGN host have a similar fraction of Lum[OIII].

Figure $6 \mathrm{c}$ shows the fraction of barred active galaxies and of AGN in the control sample, with strong $\operatorname{Lum}[\mathrm{OIII}]>10^{6.4} L_{\odot}$, as a function of the stellar age parameter $\left(D_{n}(4000)\right)$. From this figure we can see that the trend is consistent with the increase of the powerful active galaxy fraction with younger stellar population objects for both barred and unbarred AGN host galaxies. Furthermore, we can appreciate that the fraction of barred active galaxies with $\operatorname{Lum}[\mathrm{OIII}]>10^{6.4} L_{\odot}$ (solid line) is higher for younger galaxies than that of AGN hosts without bars (dashed lines). A similar trend is observed with respect to galaxy color $(g-r)$ (see Fig. 6d), where the fraction of barred active galaxies with higher nuclear activity decreases toward redder colors while the fraction of powerful unbarred AGN host remains constant for the whole $(g-r)$ range.

With the aim of understanding the behavior of the nuclear activity in AGN host galaxies with and without bars as a function of local density environments, we also measured the powerful AGN fraction with respect to the $\Sigma_{5}$ parameter. The results are shown in Fig. 6e where the fraction of powerful AGN increases slightly toward denser regions. Moreover, the significant excess of barred AGN host with high Lum[OIII] values clearly stands out with respect to the unbarred AGN host, independently of the local environment density.

\subsection{Black hole mass and accretion rate}

It is interesting to further investigate the strength of AGN. For this aim, we estimated black holes masses, $M_{\mathrm{BH}}$, for barred and unbarred AGN host galaxies, by using the observed correlation

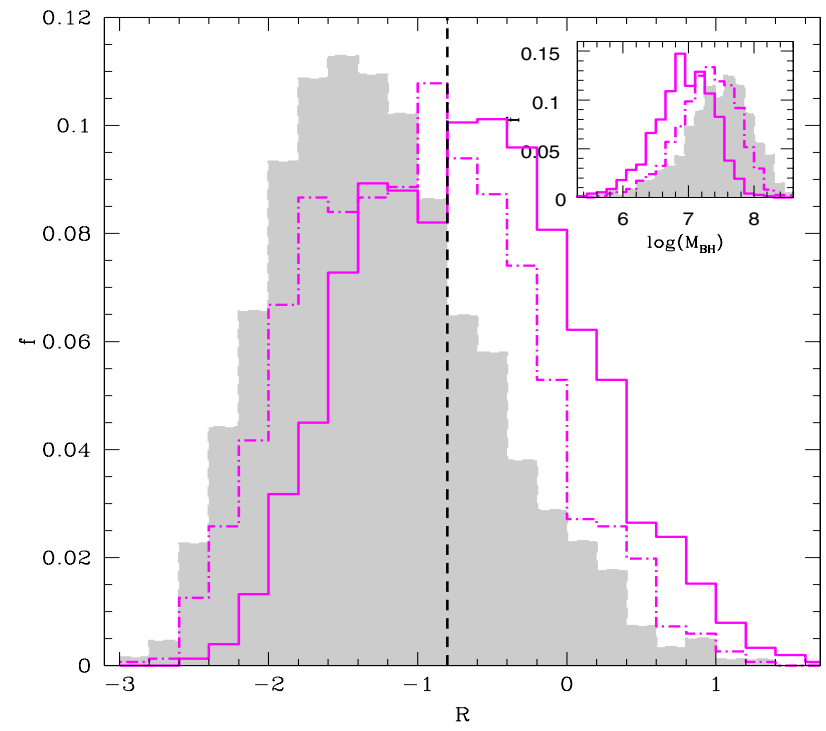

Fig. 7. Distribution of $\mathcal{R}$ for barred active galaxies (solid line) and unbarred active galaxies (full surfaces). The small box corresponds to the distribution of $\log \left(M_{\mathrm{BH}}\right)$. Dot-dashed lines represent the $\log \left(M_{\mathrm{BH}}\right)$ and $\mathcal{R}$ distributions for barred active galaxies, using the same parameters $\alpha$ and $\beta$, as for the unbarred control sample.

between $M_{\mathrm{BH}}$ and the bulge velocity dispersion $\sigma_{*}$ (Tremaine et al. 2002),

$\log M_{\mathrm{BH}}=\alpha+\beta \log \left(\sigma_{*} / 200\right)$.

The $M_{\mathrm{BH}}-\sigma_{*}$ relation is different for barred and unbarred galaxies, because the central velocity dispersion is enhanced by the stellar motion along a bar (Graham 2008). We adopted $(\alpha, \beta)=(7.67 \pm 0.115,4.08 \pm 0.751)$ for barred active galaxies and $(\alpha, \beta)=(8.19 \pm 0.087,4.21 \pm 0.446)$ for unbarred AGN host. (Gültekin et al. 2009). We restricted this analysis to active galaxies with $\sigma_{*}>70 \mathrm{~km} \mathrm{~s}^{-1}$, because the instrumental resolution of SDSS spectra is $\sigma_{*} \approx 60$ to $70 \mathrm{~km} \mathrm{~s}^{-1}$.

Furthermore, the ratio of [OIII] luminosity to black hole mass $\left(\mathcal{R}=\log \left(\operatorname{Lum}[\mathrm{OIII}] / M_{\mathrm{BH}}\right)\right)$ provides a useful measure of the accretion rate onto a black hole (Heckman et al. 2004). The big panel in Fig. 7 shows the $\mathcal{R}$ distributions for barred (solid line) and unbarred AGN host (full surface). Evidently, barred active galaxies show an excess of high accretion rate values with respect to the control sample. With the purpose of checking the effect of the bar in the active galactic nuclei in more detail, we also calculated the accretion rate for barred AGN host using the same parameters, $\alpha$ and $\beta$, that we used to obtained $\log \left(M_{\mathrm{BH}}\right)$ and $\mathcal{R}$ of the unbarred AGN host in the control sample. It is represented by dot-dashed lines in Fig. 7. Although in this last case, the signal is less significant, barred active galaxies also show an excess of accretion rate compared to the AGN without bars.

In addition, in the small box in Fig. 7, we show the distributions of black holes masses, $M_{\mathrm{BH}}$, for barred and unbarred active galaxies (solid line and full surface). This figure shows that there is a trend for $M_{\mathrm{BH}}$ to be systematically higher for unbarred AGN host. As can be observed, the accretion rate and black hole mass distributions for barred and unbarred AGN host calculated with the same parameters show a difference of lower statistical significance ( $K-S$ test with $99.95 \%$ and $98.8 \%$ confidence) than in the case when barred AGN host black hole masses are calculated with the specific $\alpha$ and $\beta$ parameters ( $K-S$ test with $100 \%$ confidence for both $\mathcal{R}$ and $M_{\mathrm{BH}}$ distributions). 
These results show that the excess of the accretion rate within a black hole in barred active galaxies with respect to unbarred AGN host is a real effect, indicating that bars play an important role in the gas infall toward the central regions in active nucleus galaxies.

\section{Summary and conclusions}

We have performed a statistical analysis of the host characteristics and nuclear activity of AGN host galaxies with and without bars. Our study is based on the sample of AGN derived from the SDSS-DR7 release, using the publicly available emission-line fluxes. We complemented the SDSS-DR7 data with the addition of a naked-eye detection of barred features of images of 6772 face-on active spiral galaxies brighter than $g$-mag $<16.5$. With the aim of providing an appropriate quantification of the effects of bars, we constructed a suitable control sample of unbarred AGN host galaxies with the same redshift, $r$-band magnitude, concentration index, bulge size parameter, and local environment distributions.

We can summarize the main results in the following conclusions.

(i) We found 1927 barred AGN host, from the full sample of 6772 spiral face-on AGN host galaxies, which represents a fraction of $28.5 \%$. This result agrees well with the bar fraction found by visual inspection of optical galaxy samples in previous works (e.g. Nilson 1973; Marinova et al. 2009; Masters et al. 2010; Oh et al. 2012) but is lower than that found using an image decomposition technique (Gadotti 2009) and near-infrared analysis images (Menéndez-Delmestre et al. 2007). We also crosscorrelated our sample with barred AGN host galaxies taken from the catalog of Nair \& Abraham (2010) and found that the visual classification of both catalogs agrees very well.

(ii) We examined the color distribution of barred and unbarred AGN host galaxies found that there is a significant excess of barred AGN hosts with red colors. The distribution of stellar population of the barred active galaxies sample shows an excess of young galaxies compared to the control sample. Redder colors and younger stellar populations found in barred active galaxies suggest that bar perturbations have a considerable effect in modifying galaxy properties in the hosts of AGN, producing a significant star formation activity and a stirring of dust in the central regions.

(iii) We also studied the fraction of galaxies with red colors and young stellar population as a function of $\log \left(M^{*}\right)$, concentration index, and local density parameter in barred active galaxies and in the control sample. We found that the number of red/young host galaxies increases/decreases with higher values of stellar mass, concentration parameter, and local environment. barred AGN host galaxies also show a higher fractions of galaxies with red colors and young stellar population, with respect to their counterpart of unbarred AGN host in the control sample.

(iv) We found that barred active galaxies show an excess of nuclear activity compared to galaxies without bars in the control sample. In addition, we found that this tendency is more significant in less massive, more concentrated light younger stellar population and bluer AGN host galaxies.

(v) We also explored the fraction of powerful AGN (with Lum[OIII] $>10^{6.4} L_{\odot}$ ) as a function of the host galaxy properties. We found that the fraction of active galaxies with $\operatorname{Lum}[\mathrm{OIII}]>10^{6.4} L_{\odot}$ increases toward more massive host with higher $C$ values, bluer colors, younger stellar population objects, and denser environments. We also found that barred AGN host objects systematically show a higher fraction of powerful AGN with respect to the control sample, in bins of different galaxy properties.

We also analyzed the accretion rate onto a black hole for barred and unbarred AGN host galaxies. From this study we conclude that barred active galaxies have an excess of objects with high accretion rate values with respect to the control sample. This result suggests that gas-rich galaxies are more efficient in accreting material toward the central region, and it also implying that bars can help in fueling material onto the central black hole.

Acknowledgements. This work was partially supported by the Consejo Nacional de Investigaciones Científicas y Técnicas and the Secretaría de Ciencia y Técnica de la Universidad Nacional de San Juan. Funding for the SDSS has been provided by the Alfred P. Sloan Foundation, the Participating Institutions, the National Science Foundation, the U.S. Department of Energy, the National Aeronautics and Space Administration, the Japanese Monbukagakusho, the Max Planck Society, and the Higher Education Funding Council for England. The SDSS Web Site is http://www.sdss.org/. The SDSS is managed by the Astrophysical Research Consortium for the Participating Institutions. The participating institutions are the American Museum of Natural History, Astrophysical Institute Potsdam, University of Basel, University of Cambridge, Case Western Reserve University, University of Chicago, Drexel University, Fermilab, the Institute for Advanced Study, the Japan Participation Group, Johns Hopkins University, the Joint Institute for Nuclear Astrophysics, the Kavli Institute for Particle Astrophysics and Cosmology, the Korean Scientist Group, the Chinese Academy of Sciences (LAMOST), Los Alamos National Laboratory, the Max-Planck-Institute for Astronomy (MPIA), the Max-Planck-Institute for Astrophysics (MPA), New Mexico State University, Ohio State University, University of Pittsburgh, University of Portsmouth, Princeton University, the United States Naval Observatory, and the University of Washington.

\section{References}

Abazajian, K. N., Adelman-McCarthy, J. K., Ageros, M. A., et al. 2009, ApJS, 182,543

Alonso, M. S., Lambas, D. G., Tissera, P. B., et al. 2007, MNRAS, 375, 1017 Athanassoula, E. 1983, IAU Symp., 100, 243

Athanassoula, E., Lambert, J., \& Dehnen, W. 2005, MNRAS, 363, 496 Baldwin, J. A., Phillips, M. M., \& Terlevich, R. 1981, PASP, 93, 5 Balogh, M., Morris, S. L., Yee, H. K. C., et al. 1999, ApJ, 527, 54 Balogh, M., Baldry, I. K., Nichol, R., et al. 2004, ApJ, 615, L101 Balzano, V. A. 1983, ApJ, 268, 602

Barrow, J. D., Bhavsar, S. P., \& Sonoda, B. H. 1984, MNRAS, 210, 19 Begelman, M. C., Blandford, R. D., \& Rees, M. J. 1980, Nature, 287, 307 Berentzen, I., Athanassoula, E., Heller, C. H., et al. 2004, MNRAS, 347, 220 Bernardi, M., Shankar, F., Hyde, J. B., et al. 2010, MNRAS, 404, 2087 Blanton, M. Rr, Eisenstein, D., Hogg, D. W., et al. 2005, ApJ, 629, 143 Bournaud, F., \& Combes, F. 2002, A\&A, 392, 83

Bournaud, F., Combes, F., \& Semelin, B. 2005, MNRAS, 364, 18 Brinchmann, J., Charlot, S., White, S. D. M., et al. 2004, MNRAS, 351, 1151

Buta, R., \& Combes, F. 1996, Fund. Cosm. Phys., 17, 95

Calzetti, D., Armus, L., Bohlin, R. C., et al. 2000, ApJ, 533, 682

Coelho, P., \& Gadotti, D. A. 2011, AJ, 743, L13

Combes, F., \& Elmegreen, B. G. 1993, A\&A, 271, 391

de Vaucouleurs, G., de Vaucouleurs A., Corwin H. G., Jr., et al. 1991, Third Reference Catalogue of Bright Galaxies (New York, NY USA: Springer) Devereux, N. A. 1987, ApJ, 323, 91

Di Matteo, T., Springel, V., \& Hernquist, L. 2005, Nature, 433, 604

Eisenstein, D. J., Annis, J., Gunn, J. E., et al. 2001, AJ, 122, 226

Ellison, S. L., Nair, P., Patton, D. R., et al. 2011, MNRAS, 416, 2182

Elmegreen, D. M., Elmegreen, B. G., \& Bellin, A. D. 1990, ApJ, 364, 415

Emsellem, E., Greusard, D., Combes, F., et al. 2001, A\&A, 368, 52

Erwin, P. 2004, A\&A, 415, 941

Gadotti, D. A. 2009, MNRAS, 393, 1531

Gadotti, D. A., \& dos Anjos, S. 2001, AJ, 122, 1298

Graham, A. W. 2008, AJ, 680, 143

Gültekin, K., Cackett, E. M., Miller, J. M., et al. 2009, ApJ, 706, 404

Gunn, J. E., Siegmund, W. A., Mannery, E. J., et al. 2006, AJ, 133, 2332

Heckman, T. M., Kauffmann, G., Brinchmann, J., et al. 2004, ApJ, 613, 109

Heckman, T. M., Ptak, A., Hornschemeier, A., et al. 2005, ApJ, 634, 161

Hernquist, L., \& Mihos, J. C. 1995, ApJ, 448, 41 
Ho, L. C., Filippenko, A. V., \& Sargent, W. L. W. 1997, ApJ, 487, 591 Kauffmann, G., Heckman, T. M., Tremonti, C., et al. 2003, MNRAS, 346, 1055 Kewley, L. J., Dopita, M. A., Sutherland, R. S., et al. 2001, ApJ, 556, 12 Kewley, L. J., Groves, B., Kauffmann, G., et al. 2006, MNRAS, 372, 961 Knapen, J. H. 2005, A\&A, 429, 141

Kuehn, F., \& Ryden, B. S. 2005, ApJ, 634, 1032

Lake, G., Katz, N., \& Moore, B. 1998, ApJ, 495, 152

Lee, G., Woo, J., Lee, M. G., et al. 2012a, ApJ, 750, 141

Lee, G.-H., Park, C., Lee, M. G., \& Choi, Y.-Y. 2012b, ApJ, 745, 125

Lintott, C., Schawinski, K., Bamford, S., et al. 2011, MNRAS, 410, 166 Lynden-Bell, D. 1969, Nature, 223, 690

Marinova, I., Jogee, S., Heiderman, A., et al. 2009, ApJ, 698, 1639

Martin, P. 1995, AJ, 109, 2428

Martin, P., \& Roy, J. R. 1994, ApJ, 424, 599

Martinez, H. J., \& Muriel, H. 2011, MNRAS, 418, 148

Masters, K. L., Nichol, R. C., Hoyle, B., et al. 2010, MNRAS, 411, 2026

Menéndez-Delmestre, K., Sheth, K., Schinnerer, E., et al. 2007, ApJ, 657, 790

Mendez-Abreu, J., Sanchez-Janssen, R., \& Aguerri, J. A. L. 2010, ApJ, 711, L61

Mihos, J. C., McGaugh, S. S., \& de Blok, W. J. G. 1997, ApJ, 477, L79

Mulchaey, J. S., Koratkar, A., Ward, M. J., et al. 1994, ApJ, 436, 586

Nair, P. B., \& Abraham, R. G. 2010a, ApJS, 186, 427
Nilson, P. 1973, Acta Universitatis Upsaliensis, Nova Acta Regiae Societatis Scientiarum Upsaliensis - Upp- sala Astronomiska Observatoriums Annaler Oh, S., Oh, K., \& Yi, S. K. 2012, ApJS, 198, 40

Perez, J., Tissera, P., \& Blaizot, J. 2009, MNRAS, 397, 748

Rees, M. J. 1984, ARA\&A, 22, 471

Roos, N. 1981, A\&A, 104, 218

Roos, N. 1985, A\&A, 294, 479

Sellwood, J. A., \& Wilkinson, A. 1993, Rep. Prog. Phys., 56, 173

Shlosman, I., Frank, J., \& Begelman, M. C. 1989, Nature, 338, 45

Shlosman, I., Begelman, M. C., \& Frank, J. 1990, Nature, 345, 679

Skibba, R. A., Masters, K. L., Nichol, R. C., et al. 2012, MNRAS, 423, 1485

Springel, V., Di Matteo, T., \& Hernquist, L. 2005, MNRAS, 361, 776

Strauss, M. A., Weinberg, D. H., Lupton, R. H., et al. 2002, AJ, 124, 1810

Tremonti, C., Heckman, T. M., Kauffmann, G., et al. 2004, ApJ, 613, 898

van den Bergh, S. 2002, AJ, 124, 782

Walker, I. R., Mihos, J. C., Hernquist, L., et al. 1996, IAU Colloq. 157, Barred Galaxies, 91, 486

Whittle, M. 1992, ApJS, 79, 49

Wyse, R. F. G. 2004, ApJ, 612, 17

Xu, C., Livio, M., \& Baum, S. 1999, AJ, 118, 1169

York, D. G., Adelman, J., Anderson, J. E. Jr., et al. 2000, AJ, 120, 1579

Zaritsky, D., Kennicutt, R. C., \& Huchra, J. P. 1994, ApJ, 420, 87. 